\title{
Fractalkine and Other Chemokines in Primary Biliary Cirrhosis
}

\author{
Shinji Shimoda, ${ }^{1}$ Carlo Selmi, ${ }^{2}$ and M. Eric Gershwin ${ }^{3}$ \\ ${ }^{1}$ Department of Medicine and Biosystemic Science, Kyushu University Graduate School of Medical Sciences, Kyushu University, \\ Fukuoka 819-0395, Japan \\ ${ }^{2}$ Division of Internal Medicine, IRCCS Istituto Clinico Humanitas, via A. Manzoni 56, Rozzano, 20089 Milan, Italy \\ ${ }^{3}$ Division of Rheumatology, Allergy and Clinical Immunology, University of California at Davis, Davis, CA 95616, USA
}

Correspondence should be addressed to Shinji Shimoda, sshimoda@intmed1.med.kyushu-u.ac.jp

Received 12 May 2011; Accepted 15 June 2011

Academic Editor: Yoshiyuki Ueno

Copyright (C) 2012 Shinji Shimoda et al. This is an open access article distributed under the Creative Commons Attribution License, which permits unrestricted use, distribution, and reproduction in any medium, provided the original work is properly cited.

\begin{abstract}
Primary biliary cirrhosis (PBC) is characterized by the autoimmune injury of small intrahepatic bile duct. On this basis, it has been suggested that the targeted biliary epithelial cells (BEC) play an active role in the perpetuation of autoimmunity by attracting immune cells via chemokine secretion. To address this issue, we challenged BEC using multiple toll-like receptor (TLR) ligands as well as autologous liver infiltrating mononuclear cells (LMNC) with subsequent measurement of BEC phenotype and chemokine production and LMNC chemotaxis by quantifying specific chemokines, specially CX3CL1 (fractalkine). We submit the hypothesis that $\mathrm{BEC}$ are in fact the innocent victims of the autoimmune injury and that the adaptive immune response is critical in PBC.
\end{abstract}

\section{Introduction}

Primary biliary cirrhosis (PBC) is a chronic cholestatic liver disease recognized at histology as chronic nonsuppurative destructive cholangitis with an autoimmune pathogenesis supported by Th1 or Th17 cells producing IFN- $\gamma$ or IL-17 $[1,2]$. Several inflammatory cell populations, including $\mathrm{T}$ and $\mathrm{B}$ cells, are found around the affected intrahepatic bile ducts, and chemokines are believed to play a pivotal role for the infiltration of inflammatory cells [3].

A better understanding of the role of specific chemokines in liver injury is ancillary to understanding the molecular mechanisms regulating the autoimmunity process and is expected to unravel new strategies to treat $\mathrm{PBC}$.

The observed patterns of chemokine expression in normal and PBC liver are illustrated in Table 1 [4]. In our'recent experiments, we cultured EpCAM-positive cells (i.e., biliary epithelial cells and BEC) isolated by immunobeads from explanted liver tissue and examined the production of chemokines by protein array following the stimulation by inflammatory cytokines or Toll-like receptor (TLR) ligands [5]. Our data illustrated that BEC produce proinflammatory chemokines such as CXCL1, CXCL5, CXCL6, and CXCL8 without any specific stimulation as shown in Figure 1. On the other hand, BEC challenged with a TLR3 ligand (poly I: C) manifest a Th1 shift and the production of CCL3, CCL4, CCL5, and CXCL10. Such production of Th1 chemokines was further prompted by the interaction between CD40 on BEC and CD154 on liver infiltrating lymphocytes. Taken altogether, the evidence support the observation that BEC induces a proinflammatory environment in the absence of innate immunity stimulation and induces Th1-sifted environment when such stimulation is present.

\section{Fractalkine}

Fractalkine is characterized as a type- 1 transmembrane molecule with the chemokine domain tethered by a 241-amino acid glycosylated stalk, a 19-amino acid transmembrane region, and 37-amino acid intracellular tail [6]. The surface-expressed transmembrane fractalkine induces the firm adhesion of leukocytes expressing its receptor CX3CR1. After shedding by the disintegrins and metalloproteinases (ADAM) 10 and 17, fractalkine also acts as a soluble leukocyte chemoattractant. Transmembrane fractalkine expressed on both endothelial and epithelial cells induces leukocyte 
Chemokine protein array from biliary epithelial cells

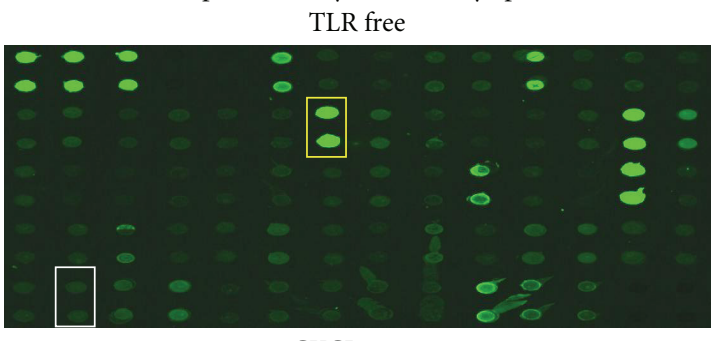

CXCL6



CXCL5

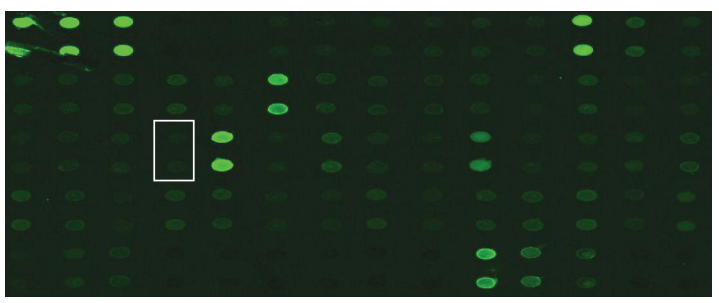

Chemokine protein array from biliary epithelial cells Poly I: C

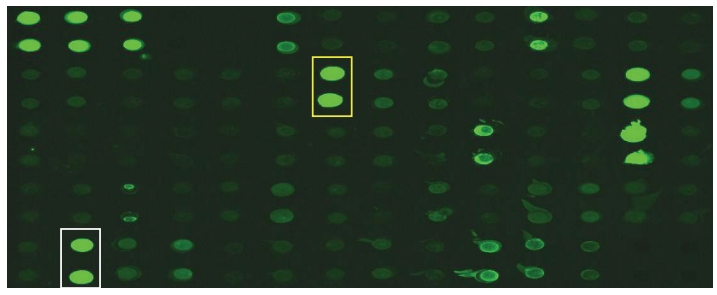

CCL5

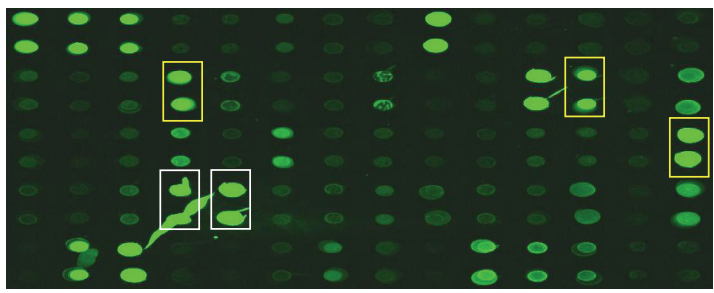

CCL3 CCL4

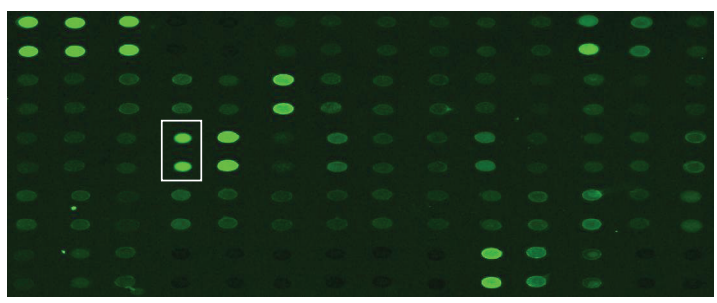

CXCL10

FIGURE 1: Chemokines produced by biliary epithelial cells under basal conditions or after stimulation with TLR3 ligand (poly I: C) for 48 hours. Cell-free culture supernatants were analyzed by a protein array kit to evaluate 174 different proteins simultaneously. Unstimulated cells produced detectable amounts of GRO- $\alpha$ /CXCL1, ENA-78/CXCL5, GCP-2/CXCL6, and IL-8/CXCL8, while poly I: C stimulation led to enhanced MIP-1 $\alpha$ /CCL3, MIP-1 $\beta /$ CCL4, RANTES/CCL5, and IP-10/CXCL10.

ELISA

Vascular endothelial cells

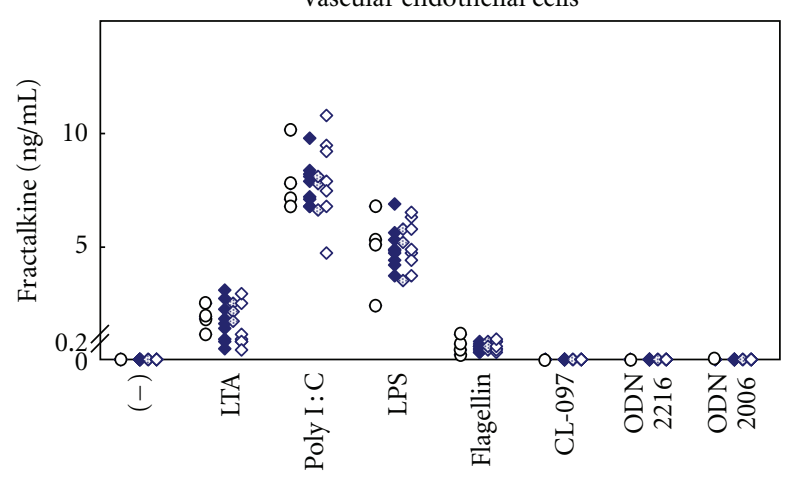

O Normal control

- $\mathrm{PBC}$ $\diamond \mathrm{HBV}$
$\diamond \mathrm{HCV}$

(a)
ELISA

Biliary epithelial cells

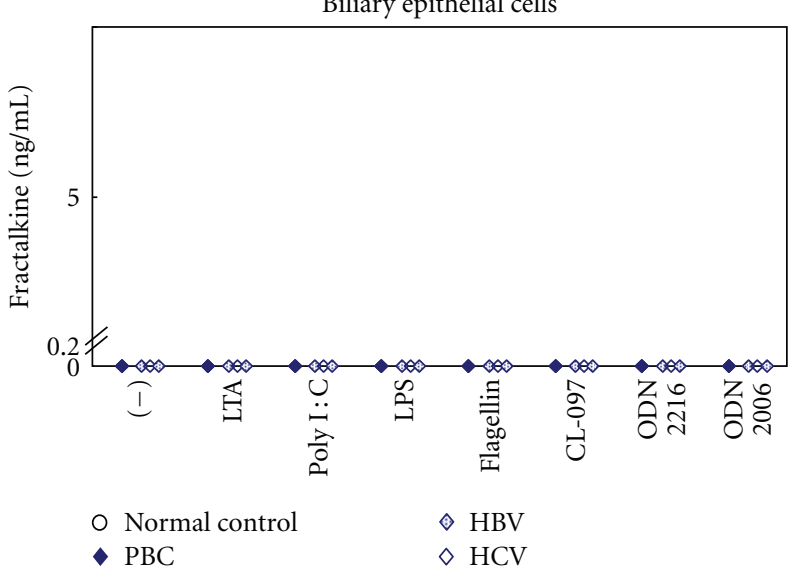

(b)

FIGURE 2: (a) Fractalkine production from endothelial cells from PBC and control (chronic hepatitis B and C) livers exposed to TLR ligands. Endothelial cells produced fractalkine with LTA, poly I : C, LPS, and flagellin with no significant differences observed between patients and control livers. (b) BEC did not produce fractalkine with any additional TLR ligand. 
TABLE 1: Chemokine expression patterns in the portal tract, sinusoidal endothelium, and bile duct of normal and PBC liver.

\begin{tabular}{|c|c|c|c|c|c|c|}
\hline \multirow{2}{*}{ Chemokine } & \multicolumn{2}{|c|}{ Portal vein } & \multicolumn{2}{|c|}{ Sinusoidal EC } & \multicolumn{2}{|c|}{ Bile duct } \\
\hline & Normal & $\mathrm{PBC}$ & Normal & PBC & Normal & $\mathrm{PBC}$ \\
\hline CXCL9 & \pm & $+(?)$ & \pm & $\mathrm{ND}$ & - & + \\
\hline CXCL10 & \pm & $+(?)$ & \pm & ND & - & + \\
\hline CXCL11 & \pm & ND & + & ND & ND & ND \\
\hline CXCL12 & - & - & - & - & + & ++ \\
\hline CXCL16 & + & + & + & + & + & ++ \\
\hline CCL25 & - & - & - & - & - & - \\
\hline CCL28 & - & + & - & - & - & ++ \\
\hline
\end{tabular}

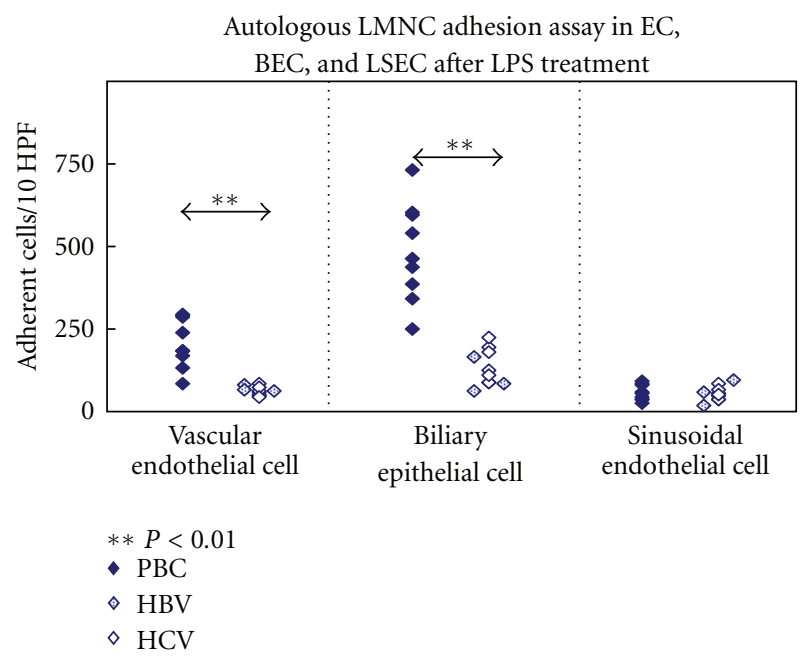

FIGURE 3: Autologous liver mononuclear cells adhesion assay using endothelial cells and BEC after stimulation with TLR4 ligand (LPS). Adherent liver mononuclear cells were stained and counted in ten random high-power microscopy fields. Liver mononuclear cells from PBC livers adhered in greater numbers than did liver mononuclear cells from controls using either endothelial cells or biliary epithelial cells, whereas liver mononuclear cells adhered only minimally to liver sinusoidal endothelial cells in all instances. Other TLR ligands did not accelerate liver mononuclear cells adhesion with neither endothelial cells nor biliary epithelial cells (data not shown).

transmigration [7]. Fractalkine is upregulated by inflammation cytokines such as TNF- $\alpha$ or IFN- $\gamma$, it has been proposed to contribute to inflammatory diseases by promoting the transmigration of CX3CR1-expressing cells to inflamed tissues in Crohn disease [8], rheumatoid arthritis, atherosclerosis [9], systemic lupus erythematosus [10], and most recently PBC [5]. CX3CR1 is expressed on natural killer cells, monocytes, macrophages, mucosal dendritic cells, $\mathrm{CD}^{+} \mathrm{T}$ cells, and a subset of effector-memory $\mathrm{CD} 4^{+} \mathrm{T}$ cells $[11,12]$. Human Th1 cells express high levels of CX3CR1 mRNA, different from polarized Th2 cells $[13,14]$. Fractalkine is expressed in limited amounts in the normal human liver, particularly near branches of the hepatic artery and in small bile ducts located at the interface between the portal tract and the hepatic lobule. In the case of acute or chronic viral hepatitis, fractalkine is detected in the areas of necrosis and inflammatory infiltration and also at the interface between the expanded portal tract and the regenerating nodule. Regenerating epithelial cells of the ductular reaction are also positive for fractalkine [15]. In kidney allograft transplantation, fractalkine is expressed in renal tubular epithelial cells, and the expression is upregulated by TNF- $\alpha$, the recognized key proinflammatory cytokine in acute rejection [16]. The $\mathrm{CD}^{+}$and $\mathrm{CD}^{+} \mathrm{T}$ cells expressing CX3CR 1 predominantly produce IFN- $\gamma$ and TNF- $\alpha$, and these T cells infiltrate the synovium in patients with rheumatoid arthritis [17]. In inflammatory bowel disease (IBD), intestinal microvascular endothelial cells produce high amounts of fractalkine, and IBD mucosa as well as periphery contained significantly more CX3CR1+ cells than control. Fractalkine is a major contributor to T- and monocytic-cell adhesion to endothelial cells [18]. In HCV infection, CX3CR1 is susceptible gene for hepatic fibrosis [19]. In mice models, it is unclear whether CX3CR1 positive cells are protective or trigger disease [20$25]$.

\section{Fractalkine and PBC}

Fractalkine is peripherally expressed dominantly in patients with $\mathrm{PBC}$, and is upregulated in $\mathrm{BEC}$ of the $\mathrm{PBC}$ liver. CX3CR1 is expressed on infiltrating lymphocytes in the portal tracts and on intraepithelial $\mathrm{T}$ cells of injured bile ducts [26]. BEC manifesting senescent features in damaged 


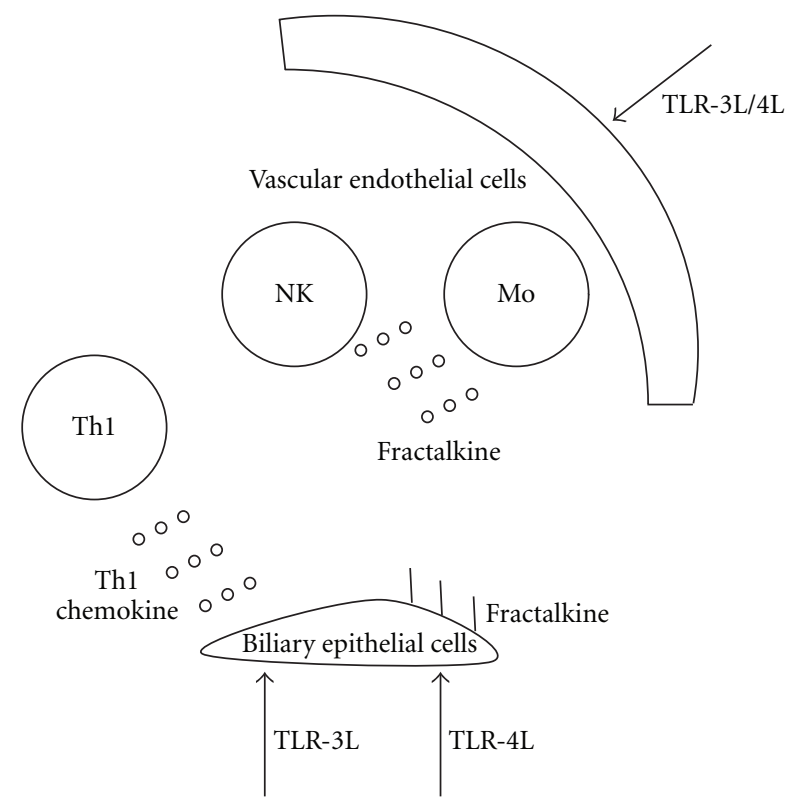

FIGURE 4: The proposed role of fractalkine is illustrated. TLR3 or TLR4 ligands stimulate vascular endothelial cells to produce fractalkine as chemokine, then fractalkine attracts CX3CR1 positive monocytes or NK cells. Subsequently, TLR4 ligand stimulated BEC produce fractalkine as cell adhesion molecule, then fractalkine recruit CX3CR1 positive cells around PBC target cells. This starts the chronic nonsuppurative destructive cholangitis and perpetuates the autoimmune pathogenesis of disease. Finally, TLR3 ligand stimulated biliary epithelial cells produce Th1 chemokines, and these chemokines are considered to contribute this autoimmune mechanism.

small bile ducts also overexpress fractalkine [27]. As previously introduced, in our recent work, we separated BEC as EpCAM positive and endothelial cells as CD31 positive by immunobeads and evaluated the production of fractalkine as chemokine by ELISA. Figure 2(a) illustrates the elevated production of fractalkine by endothelial cells challenged with TLR3 ligand (poly I : C) or TLR4 ligand (LPS). Conversely, BEC did not produce fractalkine with any other TLR ligand stimulation (Figure 2(b)), and this was not reversed with the addition of established inflammatory cytokines such as TNF- $\alpha$ or IFN- $\gamma$. Further, we investigated the production of fractalkine following the interaction between BEC or endothelial cells and liver infiltrating lymphocytes. As shown in Figure 3, mononuclear cells adhered with higher affinity to BEC compared to endothelial cells in the TLR4 ligand (LPS) stimulation, and this adherence was increased more in PBC than in other control diseases [5]. Fractalkine works to modulate inflammation in the BEC of $\mathrm{PBC}$, thus suggesting that novel therapies to block fractalkine induced environment may prove beneficial. Based on our data, we propose a working model on the role of fractalkine as chemokine or cell adhesion molecule by vascular endothelial cells and BEC, summarized in Figure 4. First, fractalkine as chemokine from vascular endothelial cells stimulated via TLR3 or TLR4 induce CX3CR1 positive monocytes or NK cells. Second, fractalkine as cell adhesion molecule from TLR4-stimulated BEC recruit CX3CR1 positive cells around target cells. This mechanism may trigger the onset of chronic nonsuppurative destructive cholangitis and autoimmune mechanism perpetuating the cholangitis. We further submit that Th1 chemokines produced by BEC stimulated from
TLR3 are important contributors to the autoimmune mechanism.

\section{References}

[1] K. Harada, J. Van De Water, P. S. Leung et al., "In situ nucleic acid hybridization of cytokines in primary biliary cirrhosis: predominance of the Th1 subset," Hepatology, vol. 25, no. 4, pp. 791-796, 1997.

[2] K. Harada, S. Shimoda, Y. Sato, K. Isse, H. Ikeda, and Y. Nakanuma, "Periductal interleukin-17 production in association with biliary innate immunity contributes to the pathogenesis of cholangiopathy in primary biliary cirrhosis," Clinical and Experimental Immunology, vol. 157, no. 2, pp. 261-270, 2009.

[3] C. T. Wu, P. A. Davis, V. A. Luketic, and M. E. Gershwin, "A review of the physiological and immunological functions of biliary epithelial cells: targets for primary biliary cirrhosis, primary sclerosing cholangitis and drug-induced ductopenias," Clinical and Developmental Immunology, vol. 11, no. 3-4, pp. 205-213, 2004.

[4] A. T. Borchers, S. Shimoda, C. Bowlus, C. L. Keen, and M. E. Gershwin, "Lymphocyte recruitment and homing to the liver in primary biliary cirrhosis and primary sclerosing cholangitis," Seminars in Immunopathology, vol. 31, no. 3, pp. 309-322, 2009.

[5] S. Shimoda, K. Harada, H. Niiro et al., "CX3CL1 (fractalkine): a signpost for biliary inflammation in primary biliary cirrhosis," Hepatology, vol. 51, no. 2, pp. 567-575, 2010.

[6] T. Imai, K. Hieshima, C. Haskell et al., "Identification and molecular characterization of fractalkine receptor CX3CR1, which mediates both leukocyte migration and adhesion," Cell, vol. 91, no. 4, pp. 521-530, 1997. 
[7] J. F. Bazan, K. B. Bacon, G. Hardiman et al., "A new class of membrane-bound chemokine with a CX3C motif," Nature, vol. 385, no. 6617, pp. 640-644, 1997.

[8] A. Muehlhoefer, L. J. Saubermann, X. Gu et al., "Fractalkine is an epithelial and endothelial cell-derived chemoattractant for intraepithelial lymphocytes in the small intestinal mucosa," Journal of Immunology, vol. 164, no. 6, pp. 3368-3376, 2000.

[9] T. Nanki, Y. Urasaki, T. Imai et al., "Inhibition of fractalkine ameliorates murine collagen-induced arthritis," Journal of Immunology, vol. 173, no. 11, pp. 7010-7016, 2004.

[10] N. Yajima, T. Kasama, T. Isozaki et al., "Elevated levels of soluble fractalkine in active systemic lupus erythematosus: potential involvement in neuropsychiatric manifestations," Arthritis and Rheumatism, vol. 52, no. 6, pp. 1670-1675, 2005.

[11] F. Geissmann, S. Jung, and D. R. Littman, "Blood monocytes consist of two principal subsets with distinct migratory properties," Immunity, vol. 19, no. 1, pp. 71-82, 2003.

[12] T. Imai, K. Hieshima, C. Haskell et al., "Identification and molecular characterization of fractalkine receptor CX3CR1, which mediates both leukocyte migration and adhesion," Cell, vol. 91, no. 4, pp. 521-530, 1997.

[13] P. Fraticelli, M. Sironi, G. Bianchi et al., "Fractalkine (CX3CL1) as an amplification circuit of polarized Th1 responses," Journal of Clinical Investigation, vol. 107, no. 9, pp. 1173-1181, 2001.

[14] M. Nishimura, H. Umehara, T. Nakayama et al., "Dual functions of fractalkine/CX3C ligand 1 in trafficking of perforin+/granzyme B+ cytotoxic effector lymphocytes that are defined by Cx3CR1 expression," Journal of Immunology, vol. 168, no. 12, pp. 6173-6180, 2002.

[15] E. Efsen, C. Grappone, R. M. DeFranco et al., "Up-regulated expression of fractalkine and its receptor CX3CR1 during liver injury in humans," Journal of Hepatology, vol. 37, no. 1, pp. 39-47, 2002.

[16] S. J. Chakravorty, P. Cockwell, J. Girdlestone, C. J. Brooks, and C. O. S. Savage, "Fractalkine expression on human renal tubular epithelial cells: potential role in mononuclear cell adhesion," Clinical and Experimental Immunology, vol. 129, no. 1, pp. 150-159, 2002.

[17] T. Nanki, T. Imai, K. Nagasaka et al., "Migration of CX3CR1positive $\mathrm{T}$ cells producing type 1 cytokines and cytotoxic molecules into the synovium of patients with rheumatoid arthritis," Arthritis and Rheumatism, vol. 46, no. 11, pp. 28782883, 2002.

[18] M. Sans, S. Danese, C. de la Motte et al., "Enhanced recruitment of CX3CR1+ T cells by mucosal endothelial cell-derived fractalkine in inflammatory Bowel disease," Gastroenterology, vol. 132, no. 1, pp. 139-153, 2007.

[19] H. E. Wasmuth, M. M. Zaldivar, M. L. Berres et al., "The fractalkine receptor CX3CR1 is involved in liver fibrosis due to chronic hepatitis C infection," Journal of Hepatology, vol. 48, no. 2, pp. 208-215, 2008.

[20] M. Inui, Y. Ishida, A. Kimura, Y. Kuninaka, N. Mukaida, and T. Kondo, "Protective roles of CX3CR1-mediated signals in toxin A-induced enteritis through the induction of heme oxygenase1 expression," Journal of Immunology, vol. 186, no. 1, pp. 423$431,2011$.

[21] T. Aoyama, S. Inokuchi, D. A. Brenner, and E. Seki, "CX3CL1CX3CR1 interaction prevents carbon tetrachloride-induced liver inflammation and fibrosis in mice," Hepatology, vol. 52, no. 4, pp. 1390-1400, 2010.

[22] C. Mionnet, V. Buatois, A. Kanda et al., "CX3CR1 is required for airway inflammation by promoting $\mathrm{T}$ helper cell survival and maintenance in inflamed lung," Nature Medicine, vol. 16, no. 11, pp. 1305-1312, 2010.

[23] S. Lee, N. H. Varvel, M. E. Konerth et al., "CX3CR1 deficiency alters microglial activation and reduces beta-amyloid deposition in two Alzheimer's disease mouse models," American Journal of Pathology, vol. 177, no. 5, pp. 2549-2562, 2010.

[24] M. Fuhrmann, T. Bittner, C. K. Jung et al., "Microglial Cx3cr1 knockout prevents neuron loss in a mouse model of Alzheimer's disease," Nature Neuroscience, vol. 13, no. 4, pp. 411-413, 2010.

[25] K. R. Karlmark, H. W. Zimmermann, C. Roderburg et al., "The fractalkine receptor CX3CR1 protects against liver fibrosis by controlling differentiation and survival of infiltrating hepatic monocytes," Hepatology, vol. 52, no. 5, pp. 1769-1782, 2010.

[26] K. Isse, K. Harada, Y. Zen et al., "Fractalkine and CX3CR1 are involved in the recruitment of intraepithelial lymphocytes of intrahepatic bile ducts," Hepatology, vol. 41, no. 3, pp. 506516, 2005.

[27] M. Sasaki, M. Miyakoshi, Y. Sato, and Y. Nakanuma, "Modulation of the microenvironment by senescent biliary epithelial cells may be involved in the pathogenesis of primary biliary cirrhosis," Journal of Hepatology, vol. 53, no. 2, pp. 318-325, 2010 . 


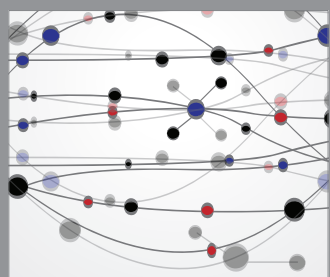

The Scientific World Journal
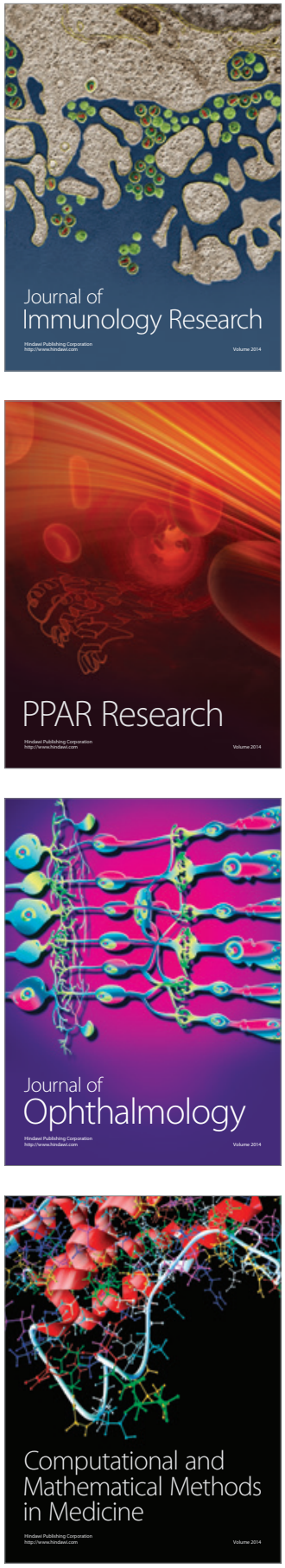

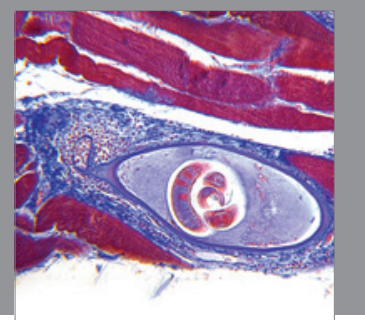

Gastroenterology

Research and Practice
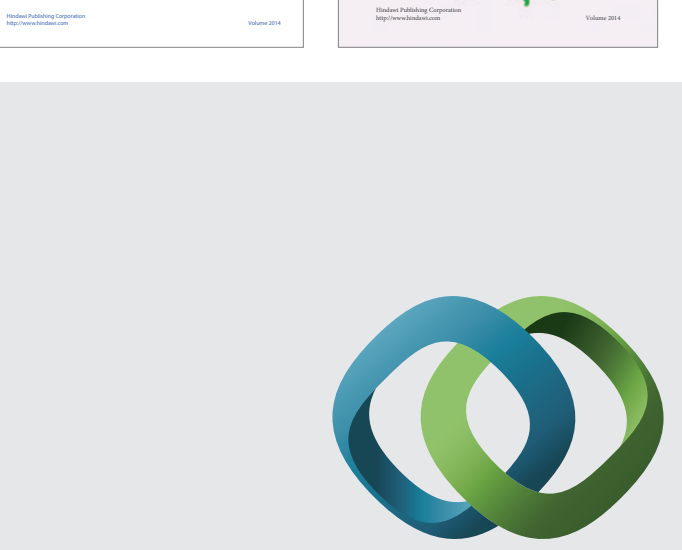

\section{Hindawi}

Submit your manuscripts at

http://www.hindawi.com
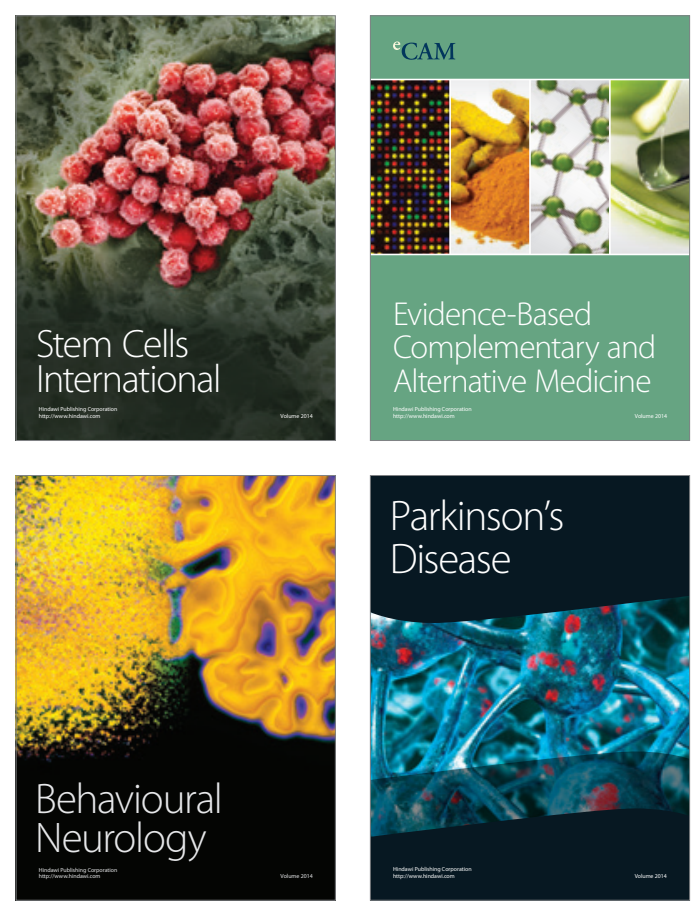

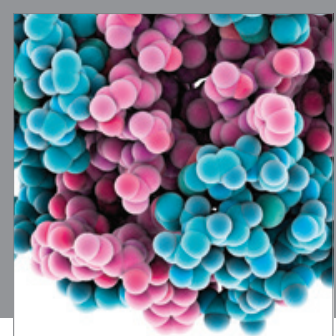

Journal of
Diabetes Research

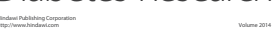

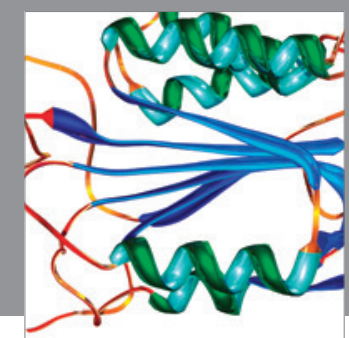

Disease Markers
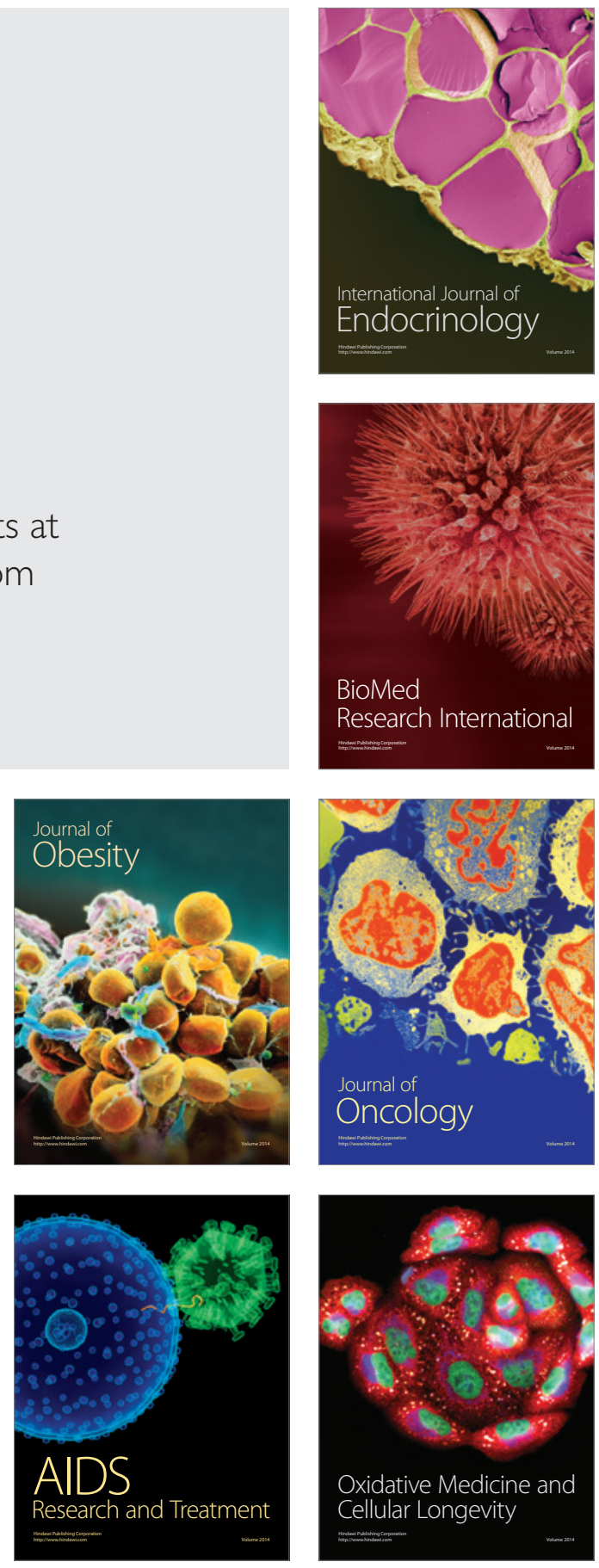